\title{
A Effectual Technique of Impulse Noise Suppression for Assessing the Impact of Brain Disorders in MRI by applying Selective Weighted Median Filter
}

\author{
${ }^{1}$ Sudheesh.K.V, ${ }^{2}$ Dr.L.Basavaraj \\ ${ }^{1}$ Research Scholar, Department of Electronics and Communication Engineering, ATME College of \\ Engineering, Mysuru, Karnataka, India \\ ${ }^{2}$ Professor, Department of Electronics and Communication Engineering, ATME College of Engineering, \\ Mysuru, Karnataka, India \\ sudheesh.vvce@gmail.com; basavaraj.atme@gmail.com
}

\begin{abstract}
An accurate analysis of medical images is progressively demanding in providing the absolute detection and diagnosis of diseases in medical imaging. The significant pre-processing step in MRI data processing is noise elimination. Noise deletion is essential step to increase image quality and performance of all the tasks desirable for quantitative imaging analysis. In this paper a new scheme for impulse noise removal in corrupted MRI brain images is introduced. The proposed scheme is a simple \& efficient filtering technique that effectively detects and removes the salt and pepper noise. The experimental results of suggested noise purifying process executed on standard set of assessment images shows that algorithm provides a very good results with low mean-squared-error and high signal-to-noise ratio values for noise density up to $95 \%$ and outperforms significant tradeoff between fine detail preservation and noise removal in brain MRI images.
\end{abstract}

Keywords: Selective weights, Denoising, Salt and pepper noise.

\section{Introduction}

In recent days, the technology of computer is extensively used in the arena of medicine. The significant area of interdisciplinary exploration is interpreting and analyzing the medical images. Many types of imaging techniques are employed for diagnosing the brain disorders in patients noninvasively without any human involvement. MRI (Magnetic Resonance Imaging) is one such advanced imaging technology which plays a vital role in creating a high quality images of human body parts, specially the parts of human brain. In image processing the two most common types of noise are Gaussian noise and Impulse noise also called as salt and pepper noise. The impulse noise in digital image is caused by malfunctioning pixels in camera sensors, defective memory cells in hardware unit or due to image transmission in noisy channel [9]. Specially, brain MRI images are more sensitive to one typical practice of impulse noise called as salt and pepper noise. Salt and pepper noise through same likelihood disturbs the quality of image by randomly corrupting the 
pixels with two extreme values 0 (black) and 255 (white) also it degrades the finest details in the medical image such as edges, textures and contours. These noises in the medical images are inevitable but removal of those noises are mandatory to boost the visual excellence of image. Thus the work focuses on exclusion of impulse noises present in the brain MRI images to realm the reliability of finest element features associated with images. Thus, in this regard the paper highly proposes the new median filtering approach based on selective weights of filter for removal of impulse noises. The performance of the denoising algorithm are evaluated using statistical noise parameters such as SNR, PSNR and MSE. The results shows that the proposed method reconstructs the high quality image to have a superior performance even at very noise density values.

\section{A new enhanced Median Filtering Approach}

Even though there are several filters available for impulse noise reduction, certain filters are best suited for some particular class of images (brain MRI Images). In most of the prevailing algorithms which includes Standard Median Filter and Center Weighted Median Filter, merely median values are used for the replacement of the degraded pixels. Therefore, in proposed algorithm we have designed improved method noise removal filter based on the statistical parameters approach in order to restore the actual input image from the degraded image. Thus, the procedure involves mainly two phases. In first phase of process, the detection of noisy pixels which is standard responsible for degradation of image is performed. The second step of process involves removal of noisy pixels and replacing those noisy pixels with noise free pixels by applying the proposed noise filtering technique. Figure 1 shows the depiction of brain MRI noisy image. In this work T2 weighted real time MRI images are used. T2 weighted images have better contrast to detect soft tissue related abnormalities which helps in classification of brain MRI images. The two sets of MRI images are collected from pathology labs a) normal images and $b$ ) abnormal images i.e. images with different abnormalities. The evaluation of proposed approach involves the T2 weighted MRI images are acquired by short echo time from Intera Philips 1.5 Tesla MRI scanner. The scanner produces the images $5 \mathrm{~mm}$ slice thickness whose resolution varies from 256X256 to 958 X958. One of the T2 weighted brain MRI image (normal and abnormal) considered in the database is shown in Figure 1.

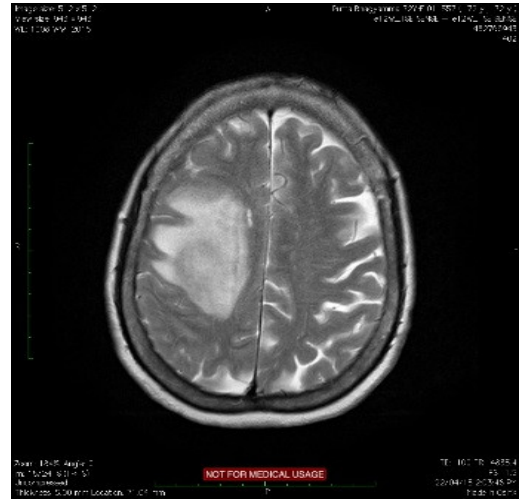

(Abnormal Image)

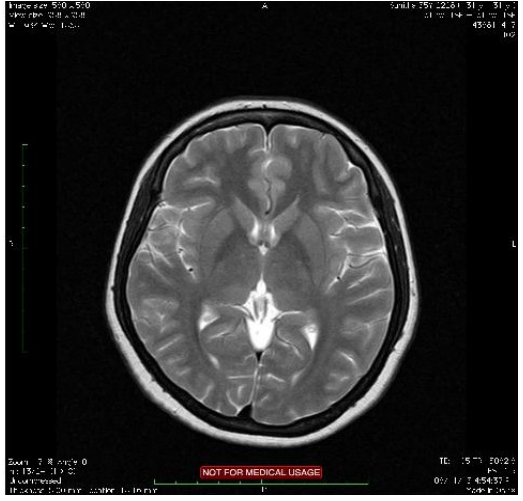

(Normal Image)

Figure 1 Original Abnormal and Normal brain MRI images 


\subsection{Noise Detection}

The most common noise present in brain MRI images is Impulse noise likewise called salt and pepper noise. A brain MRI image containing impulse noise consists of darker pixels in brighter sections and brighter pixels in darker sections. The impulse noise with same probability disturbs the quality of the image by randomly corrupting the pixels with two extreme values 0 (black) and 255 (white). The detection of impulse noise in brain MRI image can performed based on its probability density function (PDF). The PDF helps in describing the nature of the salt noise and pepper noise according to equation 1.

$$
p(k)=\left\{\begin{array}{lc}
P_{z} & \text { for } k=z \\
P_{y} & \text { for } k=y \\
0 & \text { otherwise }
\end{array}\right.
$$

Given the probability $p(k)$ ranging from 0 to 1 for that a pixel degraded, the salt and pepper noise can remain observed in an image by making probability equal to $0.5 p(z)$ by randomly selected pixels to white (salt noise) and remaining probability of $0.5 p(z)$ by randomly selected pixels to black (pepper noise).

The method for determining salt and pepper noise is also performed using morphological approach called as open close residue factors. Here, the corrupted pixels from either salt noise or pepper noise are detected by open and close residue operators. The open operator in the morphological function is responsible for determining the salty pixel and close operator is responsible for determining the pixel corrupted from pepper noise. The open and close operations on noise pixels is performed by equation 2 and 3.

$$
\begin{aligned}
& \mathrm{R}_{\mathrm{o}}(\mathrm{i}, \mathrm{j})=\mathrm{f}-\mathrm{f} \circ \mathrm{b} \\
& \mathrm{R}_{c}(\mathrm{i}, \mathrm{j})=\mathrm{f} \cdot \mathrm{b}-\mathrm{f}
\end{aligned}
$$

The corrupted pixels can be determined through equating equation 2 and equation 3 with threshold T as well-defined in equation 4.

$$
s(i, j)=\left\{\begin{array}{l}
1, R_{o}(i, j) \geq T \\
-1, R_{c}(i, j) \geq T \\
0, \text { Otherwise }
\end{array}\right\}
$$

Here, if $s(i, j)=1$, then $R_{o}(i, j)$ is determined as salt noise and if $s(i, j)=-1$, then $R_{o}(i, j)$ is determined as pepper noise. If $s(i, j)=0$, then $R_{o}(i, j)$ is taken as noise less pixel.

\subsection{Noise Removal Technique}

The work proposes a novel filtering approach called as Selective Weighted Median filter which is most effective over the Impulse (Salt and Pepper) noise commonly present within brain MRI images. The proposed filter method is variant of weighted and center weighted median filter which not solitarily considers rank command statistics nevertheless also deliberates spatial data. This filter preserves the image details by removing the additive white noise or impulse noise. It is special because of its perfectness and simplicity which means that the design of the filter is easy and the theory behind it can be easily understood. The elementary knowledge is to assign larger weights to the center pixel and the 
remaining pixels are assigned 1 in each window. The operation is same as median filter but the weights contains varying weights which is multiplied with original image pixels. The following shows the algorithm steps of the proposed filtering process.

\subsubsection{Selective Weighted Median Filtering Algorithm}

In the proposed selective weighted median filter, all the coefficients of the filter mask will have random values.

1. Consider a $3 \times 3$ mask such that highest weightage is assigned to the center pixel.

2. Next highest weightage is assigned to the four neighbors pixels of that center pixel.

3. Least weightage is assigned to the four diagonal neighbors of that center pixel.

\begin{tabular}{|l|l|l|}
\hline 1 & 1 & 1 \\
\hline 1 & 8 & 1 \\
\hline 1 & 1 & 1 \\
\hline
\end{tabular}

(Step 1)

\begin{tabular}{|l|l|l|}
\hline 1 & 4 & 1 \\
\hline 4 & 8 & 4 \\
\hline 1 & 4 & 1 \\
\hline
\end{tabular}

(Step 2)

\begin{tabular}{|c|c|c|}
\hline 1 & 4 & 1 \\
\hline 4 & 8 & 4 \\
\hline 1 & 4 & 1 \\
\hline
\end{tabular}

(Step 3)

4. Apply the $3 \times 3$ filter mask at the leftward angle of the noisy image.

5. Multiply the filter mask with the pixel value of the input noisy image.

6. After subsequent multiplication, along with center pixel arrange all four neighboring pixels of that center pixel in ascending or descending order.

7. Select the median from these five values.

8. Similarly, along with center pixel arrange all four diagonal neighboring pixels of that center pixel in ascending or descending order.

9. Select the median from these five values.

10. Replace the average of two median values at the center and move the mask throughout the noisy image and repeat the steps.

\section{Image Statistical Criteria}

The performance of the denoising algorithms are evaluated using statistical noise parameters. The statistical evaluation criteria of noises in image is used to measure the degree of improvement of the image. In this analysis the concert of every filter is calculated quantitatively for brain MRI image with impulse noise via following image evaluation criteria in directive to investigate the competence of filtering algorithms. The image statistical evaluation criteria are Signal to Noise Ratio (SNR), Peak Signal to Noise Ratio (PSNR), Structural Similarity Index (SSI), and Mean Squared Error (MSE).

\subsection{SNR Estimation}

SNR is physical degree of sensitivity of image. Larger the SNR values represents that the quality of the filtered image is noble and it is given by 


$$
S N R=10 \log _{10} \frac{\sum_{l=1}^{P} \sum_{k=1}^{Q}\left(f(l, k)^{2}+g(l, k)^{2}\right)}{\sum_{l=1}^{P} \sum_{k=1}^{Q}\left(f(l, k)^{2}-g(l, k)^{2}\right)}
$$

Where, $f(l, m)$ is original image and $g(l, m)$ is filtered image.

\subsection{PSNR Estimation}

PSNR is utmost regularly used as measure of excellence of denoised image. Greater PSNR rate designates that filtered image is of greater quality with less amount of noise. It is defined as

$$
P S N R=10 \log _{10}\left(\frac{M a x^{2}}{M S E}\right)
$$

Where, $M A X^{2}$ is the maximum intensity in the denoised images.

\subsection{SSI Estimation}

SSI is used in lieu of gaging the resemblance between output image and input image. If the enactment of filter stands effectual in eliminating impulse noises then SSI must be less than 1 and it is given by

$$
S S I=\frac{\sqrt{\operatorname{Var}(g(l, k))}}{\operatorname{Mean}(g(l, k))} X \frac{\operatorname{Mean}(f(l, k))}{\sqrt{\operatorname{Var}(f(l, k))}}
$$

\subsection{MSE Estimation}

MSE for two images [28] where one of the images is reflected as noisy estimate of the other is defined as

$$
M S E=\frac{1}{P Q} \sum_{l=1}^{P} \sum_{k=1}^{Q}(f(l, k)-g(l, k))
$$

\section{Experimental and Outcomes}

Gray-scale images of size $560 \times 560$ have been used to test the performance of the algorithm with dynamic range of values $(0,255)$. The different noise removal algorithms are tested and simulated. Denoising process is carried out for brain MRI images using the different standard impulse lessening filters such as Standard Median mesh (filter), Adaptive Median mesh, Center Weighted Median mesh, and proposed filtering approach called as Selective Weighted Median filter.

The image produced by the MRI scanners appears to be gray scale images but some images contains color bar and other details like markings and labels which are represented with different colors. Therefore it is necessary to ensure that the images are converted gray scale so that different image processing operations can be performed and the analysis becomes easier. In RGB image the corresponding intensities of red, green and blue values are the constituents of a pixel. By keeping the luminance unchanged and eliminating hue and saturation gray scale conversion is attained by 


$$
g=\left(\frac{3}{10}\right) R+\left(\frac{29}{50}\right) G+\left(\frac{11}{100}\right) B
$$

The Original image is as shown in Figure 2, and the image subjected to impulse noise (noisy image) before and after grayscale conversion is as shown in Figure 3 and Figure 4 respectively.

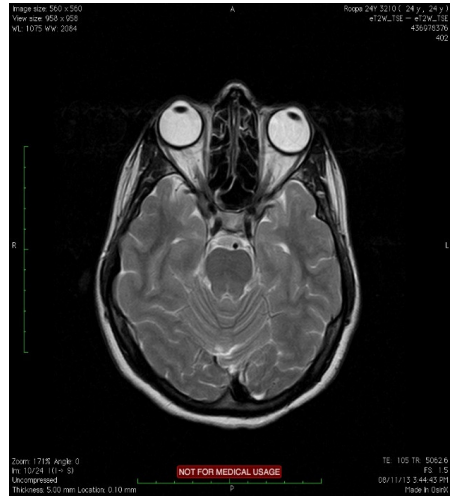

Figure 2 Original Brain MRI image

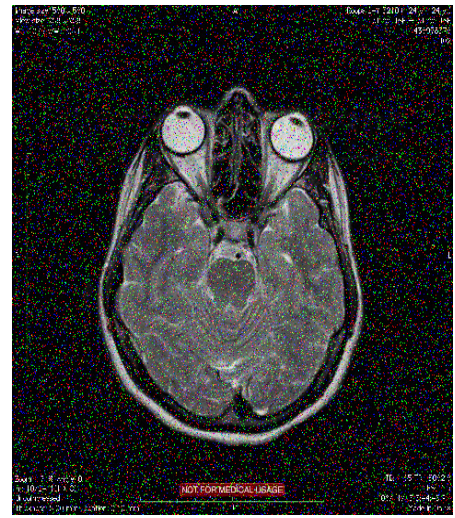

Figure 3. Noisy Image

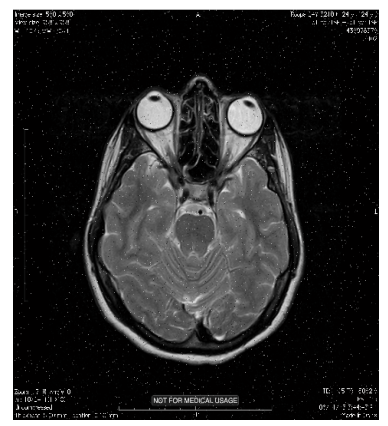

Figure 4 Noisy Image with Grayscale converted

Images will be corrupted by salt-and-pepper noise at different noise levels such as low noise level (30\%), medium noise level (60\%) and high noise level (95\%). Then the proposed filtering technique is applied to the corrupted image to remove the noise, yielding the restored gray-scale image. The denoised Image (after grayscale conversion) at different noise levels obtained after applying standard median filter, adaptive weighted median filter, and center weighted median filter and proposed selective weighted median filter for the considered example is as shown in Figure 5. The experimental tables I, II, III in this section indicates the comparative analysis of performances of different denoising median filtering algorithms at different noise levels such as low noise level (30\%), medium noise level (60\%) and high noise level (95\%) evaluated using statistical noise parameters such as PSNR, SNR, SSI and MSE. 

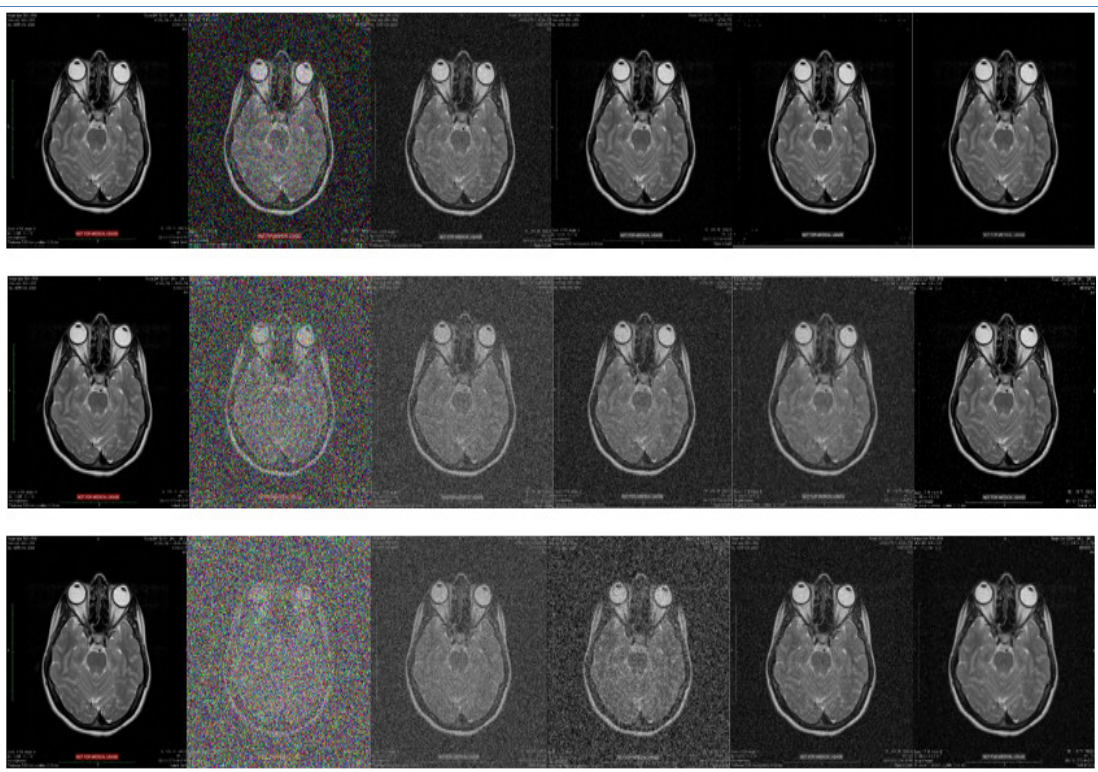
(a)
(b)
(c)
(d)
(e)
(f)

Figure 5 Simulation results of different filters on brain MRI gray-scale images. Columns represents (a) Original Image, (b) Image subjected to Impulse (salt and pepper) Noise, (c) Standard Median Filter output, (d) Adaptive Median Filter output, (e) Center Weighted Median Filter output, and column (f) represents the output of Selective Weighted Median Filter (proposed filter) output. Rows represents brain MRI image corrupted with $30 \%, 60 \%$, and $95 \%$ noise levels respectively. A comparison of images in column (b) with those in column (f) exhibits the enhanced pictorial quality of the corrupted images.

In this work, based on performance of image quality metrics the several noise removal algorithms have been tested. From Table I, II and III it is strong that proposed Selective Weighted Median filtering approach eliminates the noise to the maximum extent since it provides the lowest Mean Square Error (MSE) and very extraordinary Peak Signal to Noise Ratio (PSNR) values for noise level ranging between $90 \%$ to $95 \%$. Thus the proposed approach emerge as a best and promising noise removal technique which outperforms the conventional steps for removal of substantial amount of noise present in brain MRI images and helping in preserving the edges and finest details.

Table 1: Comparative analysis of different noise removal algorithms at low noise level of $30 \%$

\begin{tabular}{|l|c|c|c|c|c|}
\hline \multirow{2}{*}{ Filtering Methods } & \multicolumn{4}{|c|}{ Image Statistical Criterions at low Noise Level = 30\% } \\
\cline { 2 - 6 } & PSNR & SNR & SSI & MSE & Time \\
\hline Selective Weighted Median Filter & 32.1812 & 17.2205 & 0.9808 & 3.0708 & 1.21 \\
\hline Center Weighted Median Filter & 27.1089 & 14.1385 & 0.9761 & 9.4437 & 2.16 \\
\hline Adaptive Median Filter & 26.3591 & 12.3910 & 0.8990 & 13.8981 & 1.15 \\
\hline Standard Median Filter & 21.2810 & 10.3083 & 0.8762 & 15.2896 & 1.13 \\
\hline
\end{tabular}

Table 2: Comparative analysis of different noise removal algorithms at low noise level of $60 \%$

\begin{tabular}{|l|c|c|c|c|c|}
\hline \multirow{2}{*}{ Filtering Methods } & \multicolumn{5}{|c|}{ Image Statistical Criterions at Medium Noise Level = 60\% } \\
\cline { 2 - 6 } & PSNR & SNR & SSI & MSE & Time \\
\hline Selective Weighted Median Filter & 24.3603 & 8.8675 & 0.5015 & 19.6176 & 1.20 \\
\hline Center Weighted Median Filter & 22.8054 & 7.6578 & 0.3901 & 28.5657 & 2.16 \\
\hline Adaptive Median Filter & 20.1378 & 5.0704 & 0.5127 & 41.6680 & 1.15 \\
\hline Standard Median Filter & 18.7430 & 4.3803 & 0.2007 & 47.2475 & 1.12 \\
\hline
\end{tabular}


Table 3: Comparative analysis of different noise removal algorithms at low noise level of $95 \%$

\begin{tabular}{|l|c|c|c|c|c|}
\hline \multirow{2}{*}{\multicolumn{1}{|c}{ Filtering Methods }} & \multicolumn{4}{|c|}{ Image Statistical Criterions at High Noise Level = 95\% } \\
\cline { 2 - 6 } & PSNR & SNR & SSI & MSE & Time \\
\hline Selective Weighted Median Filter & 19.5222 & 5.7716 & 0.3561 & 27.2482 & 1.21 \\
\hline Center Weighted Median Filter & 17.2665 & 4.2278 & 0.2703 & 37.5925 & 2.21 \\
\hline Adaptive Median Filter & 16.7041 & 3.5551 & 0.1299 & 66.4134 & 1.18 \\
\hline Standard Median Filter & 15.9743 & 3.1164 & 0.0925 & 74.2245 & 1.07 \\
\hline
\end{tabular}

The Figures 6 - 9 demonstrates the contrast of different filters (SWMF, CWMF, AMF and SMF) performed on brain MRI image at various noise densities, in terms of PSNR, SNR, SSIM, and MSE.

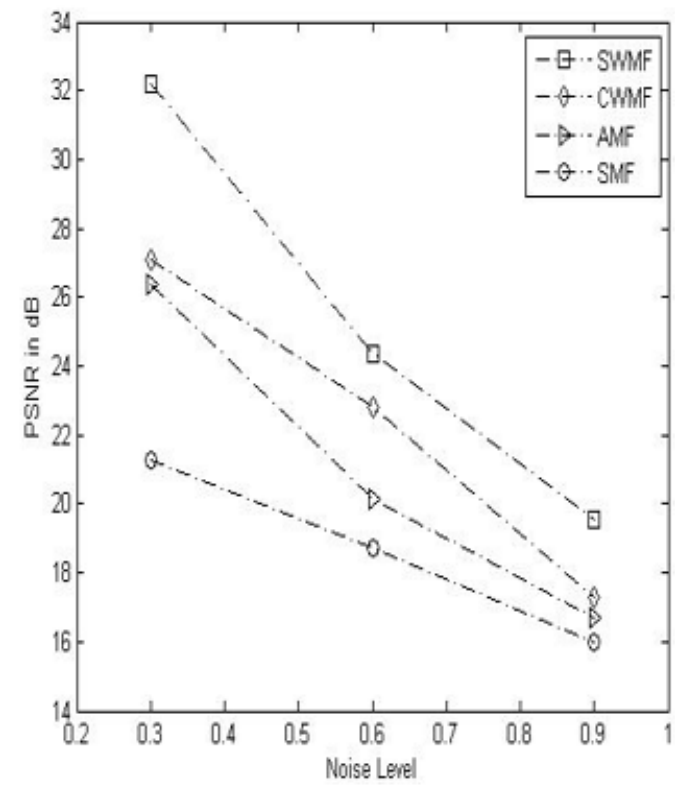

Figure 6 PSNR in dB v/s Noise Level

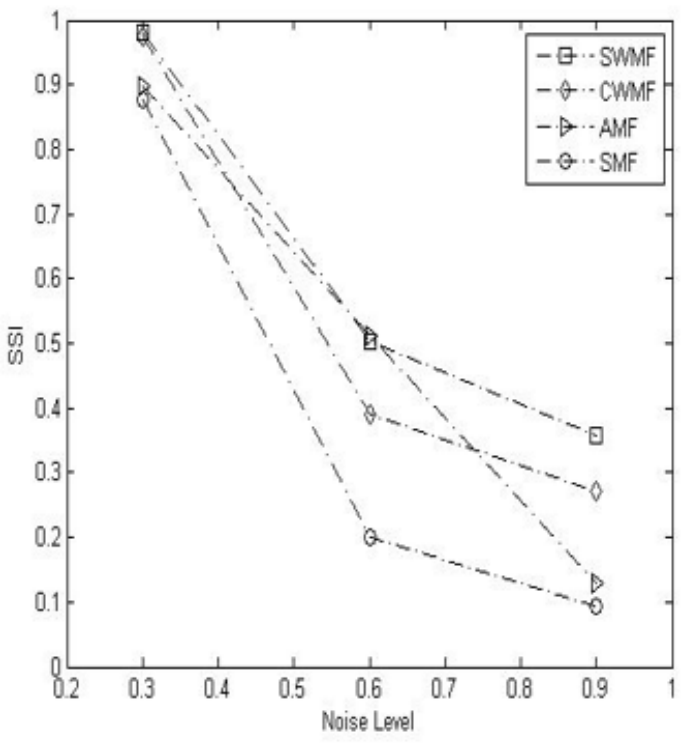

Figure 8. PSNR in dB v/s Noise Level

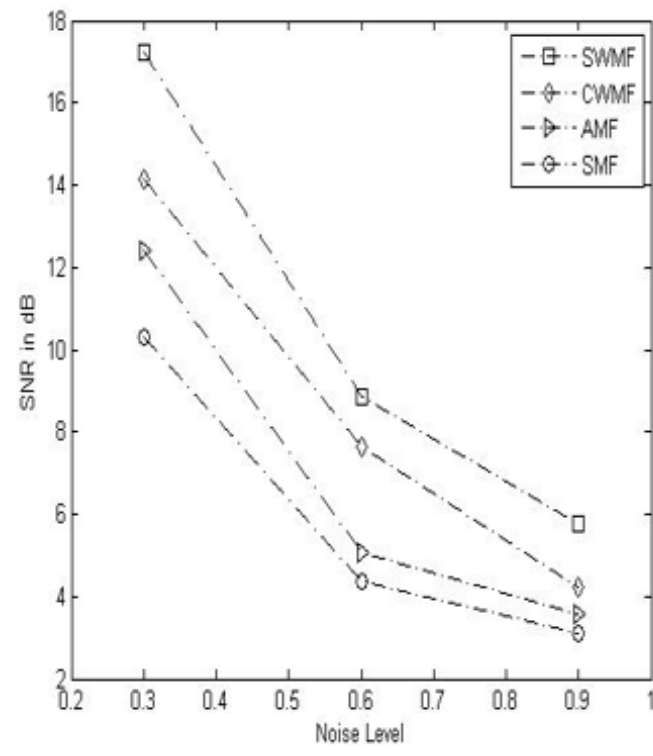

Figure 7 SNR in dB v/s Noise Level

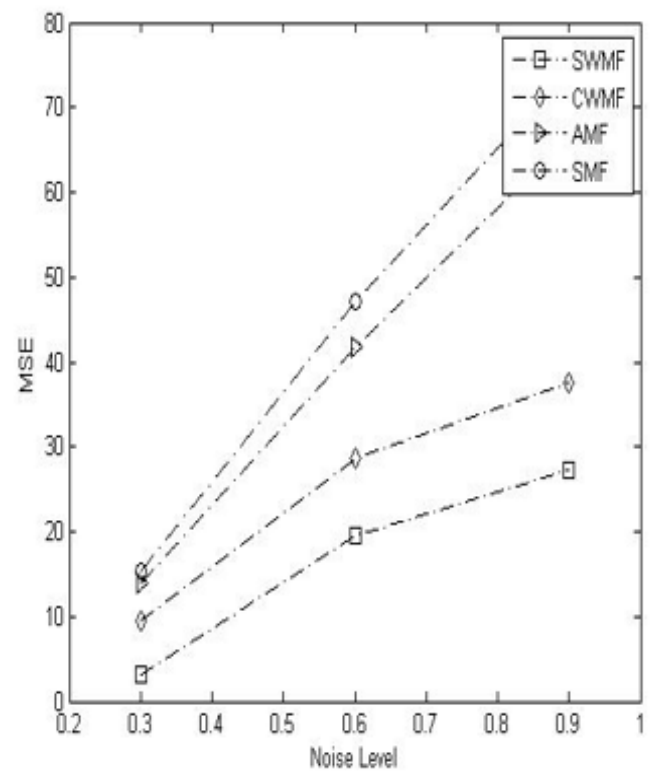

Figure 9. SNR in dB v/s Noise Level 


\section{Conclusion}

The objective of the work is accomplished by designing and implementing the current techniques for deduction of impulse (salt and pepper) noises present in brain MRI images by providing simple and best noise filtering algorithm. In this paper we have subjected the T2 weighted real time MRI images of brain for estimating and suppressing the impulse noise using a proposed novel approach of filtering called as Selective Weighted Median filter. This work includes subsequent preprocessing steps of noise detection, grayscale conversion, and noise removal in brain MRI images. The comparative analysis of various noise removal algorithms shows that proposed algorithm provides a good results with high PSNR, SNR and low MSE values for the noise level nearly up to 95\%. The results are obtained based on image evaluation criteria which is evaluated using noise statistical parameters such as PSNR, SNR, SSI and MSE. The proposed work has achieved remarkable results which helps in choosing the accurate filtering technique. And the effort can be further studied to enhance the results even when noise level increases beyond $95 \%$ by preserving the finest details very well.

\section{REFERENCES}

[1] M. a. Yousuf and M. N. Nobi, "A New Method to Remove Noise in Magnetic Resonance and Ultrasound Images," J. Sci. Res., vol. 3, no. 1, 2011.

[2] Chan, R.H., Ho, C.W., and Nikolova, M, "Salt-and-pepper noise removal by median-type noise detectors and detail-preserving regularization," IEEE Trans. Image process, pp. 1479-1485, 2005.

[3] Madhu S. Nair, K. Revathy, and Rao Tatavarti, "Removal of Salt-and Pepper Noise in Images: A New Decision-Based Algorithm", Proceedings of the International Multi Conference of Engineers and Computer Scientists 2008 Vol I IMECS 2008, 19-21 March, 2008, Hong Kong.

[4] Sajjad Mohsin, Sadaf Sajjad, Zeeshan Malik, and Abdul Hanan Abdullah, "Efficient Way of Skull Stripping in MRI to Detect Brain Tumor by Applying Morphological Operations, after Detection of False Background", International Journal of Information and Education Technology, Vol. 2, No. 4, August 2012.

[5] K. S. Srinivasan, D. Ebenezer, "A New Fast and Efficient Decision- Based Algorithm for Removal of HighDensity Impulse Noises," IEEE Signal Processing Papers, Vol. 14, No. 3, pp. 189-192, March 2007.

[6] H. Hwang and R. A. Haddad, "Adaptive median filters: New algorithms and results," IEEE Trans. Image Process., Vol. 4, No. 4, pp. 499-502, Apr. 1995.

[7] Geoffrine Judith.M.C, and N.Kumarasabapathy. "Study and analysis of impulse noise reduction filters", Signal \& Image Processing: An International Journal (SIPIJ), Vol.2 (1), pp.82-92, 2011.

[8] Anjanappa C and Sheshadri H.S., "Comparative Analysis of Efficient Impulse Noise Removal Techniques applied to Medical Images based on Mathematical Morphology", International Research Journal of Medical Sciences ISSN 2320 -7353 Vol. 3(9), 1-12, September (2015). 
[9] B. Kwan and H. Kwan, "Impulse noise reduction in brain magnetic resonance imaging using fuzzy filters," World Academy of Science, Engineering Technology, vol. 60, pp. 1194-1197, 2011.

[10] E. George and M. Karnan, "MRI Brain Image Enhancement Using Filtering Techniques", International Journal of Computer Science \& Engineering Technology (IJCSET), ISSN: 2229-3345 Vol. 3 No. 9 Sep 2012.

[11] K. Somasundaram, T.Kalaiselvi, "Automatic brain extraction methods for T1 magnetic resonance images using region labeling and morphological operations", Computers in Biology and Medicine 41 (2011) Elsevier Ltd, pp. 716-725

[12] Chih-Yuan Lien, Chien-Chuan Huang, Pei-Yin Chen, Yi-Fan Lin, "An Efficient Denoising Architecture for Removal of Impulse Noise in Images", IEEE Transactions on Computers, vol.62, no. 4, pp. 631-643, April 2013, doi:10.1109/TC.2011.256

[13] I. Shanthi, Dr. M.L. Valarmathi, "Speckle Noise Suppression of SAR Color Image using Hybrid Mean Median Filter", International Journal of Computer Applications (0975 - 8887) Volume 31- No.9, October 2011

[14] Anisha K K and Dr.M.Wilscy, "Impulse Noise Removal from Medical Images Using Fuzzy Genetic Algorithm", The International Journal of Multimedia \& Its Applications (IJMA) Vol.3, No.4, November 2011.

[15] Jin Hyuk Hong, Sung Bae Cho and Ung Keun Cho (2009) "A Novel Evolutionary Method to Image Enhancement Filter Design: Method and Applications", IEEE Transactions on Systems, Man and Cybernetics - Part B, Cybernetics, Vol. 39, No. 6, pp. 1446-1457.

[16] Sung JeaKo and Yong Hoon Lee (1991) "Center Weighted Median Filters and their application to Image Enhancement", IEEE Transactions on Circuits and Systems, Vol 38, No 9.

[17] Chen C., Wng J., Quin W. and Dong X., "A new adaptive weight algorithm for Salt and Pepper noise removal", Consumer Electronics, Communication and Networks, pp.26-29, 2011.

[18] Kenny K.V. Toh and Nor A.M. Isa., "Noise adaptive fuzzy switching median filter for Salt-and- Pepper noise reduction", IEEE signal processing letters, Vol.17 (3), pp.281-284, 2010

[19] M.Ramesh, P.Priya, Punal.M.Arabi, "A Novel Approach for Efficient Skull Stripping Using Morphological Reconstruction and Thresholding Techniques", International Journal of Research in Engineering and Technology eISSN: 2319-1163 | pISSN: 2321-7308, Volume: 03 Issue: 01 Jan-2014

[20] K.Somasundaram, and R.Siva Shankar, "Skull stripping based on clustering and curve fitting with quadratic equations", ICMMSC, CCIS 283, PP 439-444. Springer - Verlag Berling Heidelberg 2012.

[21] R.C.Gonzales amd R.E.Woods, Digital image processing, Second edition, Prentice Hall, 2002.

[22] Rosniza Roslan, Nursuriati Jamil and Rozi Mahmud, "Skull Stripping Magnetic Resonance Images Brain Images: Region growing versus Mathematical Morphology", International Journal of Computer Information Systems and Industrial Management Applications, Vol 3, pp 150-158, 2011. 